\title{
Effects of Excitotoxin Exposure on Metabolic Rate of Primary Hippocampal Cultures: Application of Silicon Microphysiometry to Neurobiology
}

\author{
Kathleen M. Raley-Susman,' Karen R. Miller, ${ }^{2}$ John C. Owicki, ${ }^{2}$ and Robert M. Sapolsky' \\ 'Department of Biological Sciences, Stanford University, Stanford, California 94305 and ${ }^{2}$ Molecular Devices Corporation, \\ Menlo Park, California 94025
}

Increasing evidence implicates glutamate receptor overstimulation in the neurotoxicity associated with a host of metabolic insults, including seizures and hypoxia-ischemia. To begin to understand more completely the role of energy metabolism in the mechanism of neuron death following excitatory amino acid exposure, we investigated the effects of kainic acid exposure on metabolic rate in cultured hippocampal cells using a recently developed silicon microphysiometer. The device gives a continual real-time measure of metabolism in relatively small numbers of cells, as assessed by efflux of protons generated at least in part by ATP hydrolysis and lactic acid production. In the first half of this report, we characterize the feasibility of using this device for measuring cellular metabolism in hippocampal cultures. Metabolic rate in both astrocytes and neurons was readily detectable, with a high signal-to-noise ratio. The rate was proportional to the number of cells and was sensitive to metabolic enhancement or depression. We then utilized this device to study metabolic responses to the excitotoxin kainic acid. We observed a receptor-mediated, dose-dependent increase in metabolic rate upon stimulation by kainic acid, with an $E_{50}$ of $\sim 100 \mu \mathrm{M}$. Exposure to toxic levels of kainic acid for 10 min produced an initial elevation (for $2 \mathrm{hr}$ ) in metabolic rate and then a gradual decline in metabolism over the next $8 \mathrm{hr}$ that preceded a measurable loss of cell viability. This study further delineates a time window for the onset of kainic acid-induced damage. The results clearly show the feasibility of using silicon microphysiometry for assessing metabolism of brain cultures and for exploring the relationship between metabolism and synaptic activation.

Activation of glutamate receptors is thought to stimulate neuronal energy consumption for the operation of the cells' energy-dependent ion homeostatic mechanisms. However, the metabolic consequences of glutamate exposure under normal conditions are not well understood, largely because direct measurement of

Received May 28, 1991; revised Oct. 1, 1991; accepted Oct. 7, 1991.

This work was supported by an American Heart Association, Santa Barbara Chapter, postdoctoral fellowship (K.M.R.-S.) and by NIH Grant R01-AG06633 (R.M.S.). Partial support was also provided by U.S. Army CRDEC and DARPA programs (to Molecular Devices, Inc.). We thank G. Tombaugh and Dr. L. Jacobson for helpful comments about the manuscript, and the members of the Sapolsky lab for assistance with tissue culture procedures.

Correspondence should be addressed to Dr. Kathleen M. Raley-Susman, Department of Biology, Vassar College, Poughkeepsie, NY 12601.

Copyright (c) 1992 Society for Neuroscience $0270-6474 / 92 / 120773-08 \$ 05.00 / 0$ metabolic rate in healthy brain tissue is technically difficult. For example, while in vivo studies have measured metabolic parameters, such as ${ }^{14} \mathrm{C}$-2-deoxyglucose uptake (Cremer et al., 1988) (which affords excellent cellular resolution), the time resolution necessary for responses to receptor activation is quite poor. Further, studies with brain slices measuring oxygen consumption in response to bath application of glutamate receptor agonists (Nishizaki et al., 1988) suffer from limitations relating to the diffusion of oxygen from the center of tissue whose edges are damaged. These technical difficulties also limit the ability to measure directly the effects of toxic concentrations of excitatory amino acids such as glutamate. This determination is important because it is generally thought that exposure to excitatory amino acids is damaging when energy stores are excessively taxed because of overstimulation of receptors (Choi, 1988; Novelli et al., 1988). This excitotoxicity hypothesis rests on the assumption that the mechanism of neuron damage is energetic in nature, and yet few direct assessments of the metabolic effects of excitotoxin exposure have been undertaken to test this assumption.

In the present study, we measured the metabolic rate in primary hippocampal cultures in response to kainic acid using a silicon microphysiometer, a novel device that allows for realtime, sensitive measurement of cellular metabolism (Parce et al., 1989; Owicki et al., 1990). The silicon microphysiometer, a semiconductor-based instrument, detects the extrusion of acidic metabolic products of glycolysis, respiration, and ATP hydrolysis (Parce et al., 1989), including lactic acid, $\mathrm{CO}_{2}$, and protons. In addition, the device may detect transient proton fluxes such as those caused by imbalances between ATP synthesis and hydrolysis, or by changes in intracellular pH (Owicki and Parce, in press). Previous work has demonstrated the usefulness of the microphysiometer for screening cellular responses to therapeutic drugs, growth factors, and hormone/neurotransmitter receptors transfected into cell lines (Parce et al., 1989; Owicki et al., 1990). The present report demonstrates the usefulness of using silicon microphysiometry for studying neurotransmitter receptor activation in primary neuronal cultures and demonstrates a correlation between neurotransmitter exposure and neuronal metabolism.

We show that the microphysiometer is sensitive to the metabolic status of hippocampal cultures. In addition, we observe that acute exposure to kainic acid increases metabolic rate in a receptor-specific, dose-dependent manner. Further, we observe that toxic concentrations of kainic acid cause a delayed decline in metabolic rate that precedes cell death. 


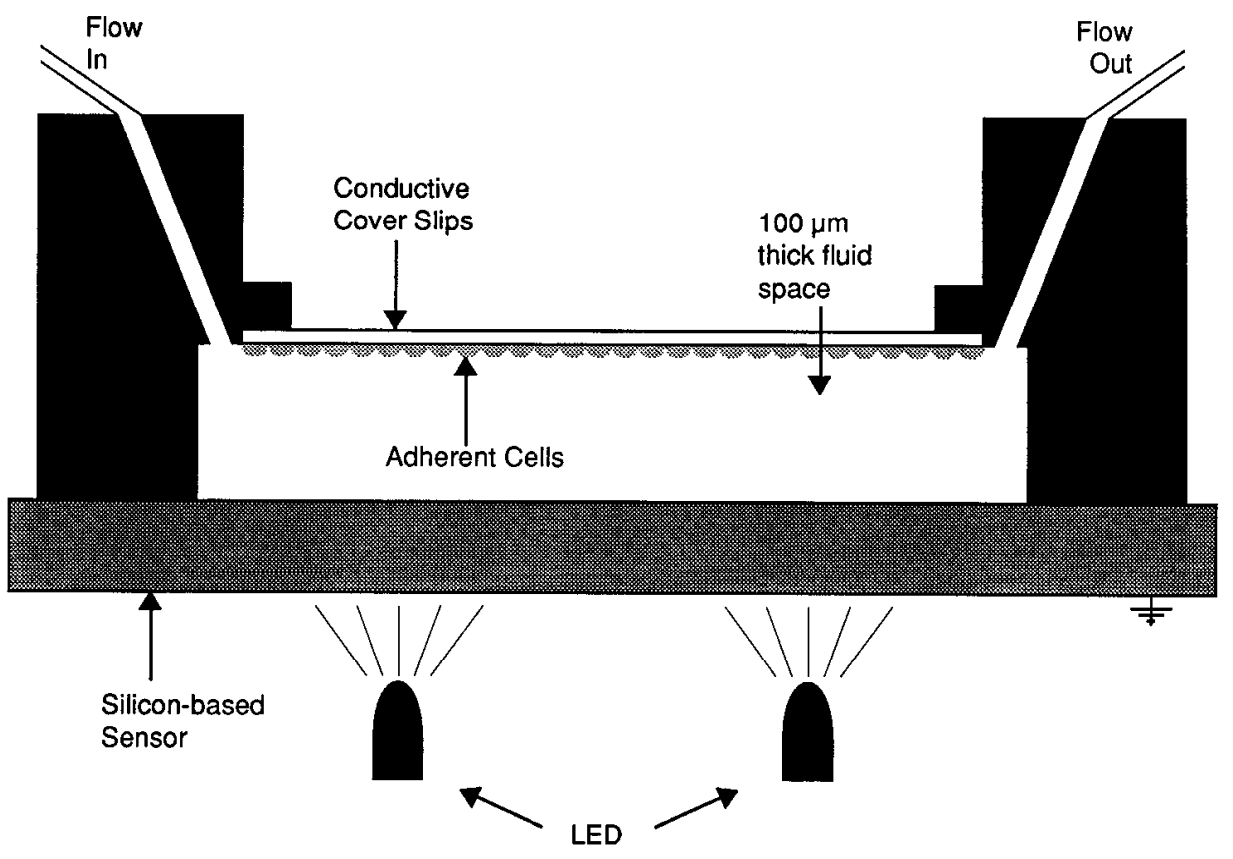

Figure 1. Schematic diagram of cellcontaining chamber. Metabolic rate determinations are obtained from cells directly above the region illuminated by the LED (see text). Perfusion of experimental medium is under computer control. The silicon-based sensor forms the bottom of the cell chamber, while the conductive coverslip forms the top of the chamber.

\section{Materials and Methods}

Buffers. Cell cultures were maintained in Dulbecco's modified Eagle's medium (DMEM; GIBCO, Grand Island, NY) containing $5.55 \mathrm{~mm}$ glucose, $10 \%$ fetal calf serum (FCS; GIBCO), and $1 \%$ penicillin, $1 \%$ streptomycin (GIBCO). Twenty-four hours prior to some experiments (see Results), the medium was changed with two washes of DMEM (Sigma) containing the concentrations of glucose indicated in Results. For metabolic rate determinations, microphysiometer perfusates consisted of a modified DMEM lacking bicarbonate to reduce the buffering capacity of the extracellular medium to $1 \mathrm{~mm}$ (Owicki et al., 1990). The concentrations of glucose and other constituents are as indicated in Results.

Culture preparation. Cultured hippocampal neurons were isolated according to Banker and Cowan (1977) and prepared as described previously (Sapolsky et al., 1988) with modifications. Briefly, 50-100 hippocampi from day 18 fetal rats were dissected from the brain, washed, and enzymatically and mechanically dispersed with $0.05 \%$ trypsin (GIB$\mathrm{CO}$ ) or $0.4 \%$ collagenase II (Worthington Biochemicals, Freehold, NJ) and DNase II (Sigma) in 0.1\% BSA (Calbiochem) in HEPES-buffered Hank's balanced salt solution (GIBCO). Cells were plated to a density of approximately $1-2 \times 10^{5} \mathrm{cells} / \mathrm{cm}^{2}$ and maintained in high-glucose HEPES-buffered DMEM (GIBCO) with 5\% FCS (IIyclone Laboratories, Logan, UT). The cells were grown on poly-D-lysine-coated conductive glass coverslips. The coverslips were coated on the cellular side with indium-tin oxide to provide a conductive surface. The coating did not affect the adherence properties or the viability of the cultures. Cultures were maintained for $10 \mathrm{~d}$ in DMEM (with $5 \mathrm{~mm}$ glucose) with $5 \%$ FCS at $37^{\circ} \mathrm{C}$ in an incubator equilibrated with $10 \% \mathrm{CO}_{2}$. Cells were refed on day 5 with fresh medium containing $30 \mu \mathrm{g} / \mathrm{ml}$ uridine (Sigma) and 15 $\mu \mathrm{g} / \mathrm{ml}$ fluoro-deoxyuridine (Sigma) to retard glial proliferation.

At the time of experimentation (10-15 d), mixed cultures consisted of 50-60\% glia, which form a flat, discontinuous monolayer of polygonal cells along the bottom of the coverglass, and $40-50 \%$ neurons, which are phase bright and overlie the glial monolayer. Morphological criteria for neurons and glia were determined previously based on immunocytochemical analysis with antisera to neuron-specific enolase and glial fibrillary acidic protein (Horner et al., 1990). As noted above, $24 \mathrm{hr}$ prior to experimentation, cells were pretreated with DMEM lacking serum and containing different concentrations of glucose.

Glia-enriched cultures were obtained by omitting the mitotic inhibitors and refeeding the cultures every $3-4 \mathrm{~d}$ for 4 weeks. This treatment resulted in a confluent layer of flat, polygonal-shaped cells and no overlying phase-bright neuronal cells.
Measurement of cellular metabolic rate. Metabolic rates of hippocampal mixed cultures were measured using the silicon microphysiometer, as has been described in detail elsewhere (Parce et al., 1989; Owicki et al., 1990). Briefly, coverslips containing cells were placed in a lowvolume flow chamber, one side of which is a silicon-based light-addressable potentiometric sensor that measurcs small changes in extracellular medium $\mathrm{pH}$. The other side of the chamber is the cell-bearing coverslip, which is coated with indium-tin oxide to create a conductive surface. Cultures were perfused with a low-buffering-capacity (1 mM) medium at $15 \mu \mathrm{l} / \mathrm{min}$ for $150 \mathrm{sec}$, followed by a $100 \mathrm{sec}$ period of halted flow. The perfusion/halt cycle was controlled by an IBM PC interface.

Metabolic rate was determined as the rate of acidification of the external medium during the brief halt $(100 \mathrm{sec})$ in the perfusion. Previous studies have shown that the rate of acidification of the external medium is a sensitive index of metabolic rate in various cell types (Parce et al., 1989) and correlates well with other indices of metabolism, including lactate production and oxygen consumption. Acidification rates of the medium were measured at regions of the silicon/electrolyte interface using a light-emitting diode (Fig. 1). The rate of acidification was determined as the slope of a linear least-squarcs fit to the relation of $\mathrm{pH}$ versus time (sec). The perfusion then resumed, allowing the $\mathrm{pH}$ of the medium to return to basal levels (within 10-15 sec of perfusion onset). Multiple determinations of the acidification rate were obtained, and the data are expressed as the average of 5-10 such determinations at a given time point (see Results).

In some experiments, we used a version of the microphysiometer that utilized an He-Ne laser beam, projected through the optics of a microscope (Parce et al., 1989), to illuminate a total sensing region of $1 \mathrm{~mm}^{2}$ of the silicon light-addressable sensor. This setup allowed us to select fields with particular cell densities and that were enriched with particular cell types (astrocytes or neurons)

Drug treatments. As indicated above, for experiments investigating the effects of substrate availability on basal metabolic rate, cultures were pretreated $24 \mathrm{hr}$ prior to experimentation with the indicatcd glucose concentration. The effects of cyanide or kainic acid on metabolic rate were studied by injecting the indicated solutions into the tubing leading to the cell chambers on the microphysiometer. All solutions were warmed to $37^{\circ} \mathrm{C}$ and adjusted to $\mathrm{pH} 7.4$ with $\mathrm{NaOH}$ or $\mathrm{HCl}$. Cells were exposed to the solutions for the times indicated in Results.

$L D H$ assay. At the conclusion of some experiments, coverslips were removed from the microphysiometer and placed in lysis buffer $(0.1 \%$ Triton $\mathrm{X}-100$ ) overnight at $4^{\circ} \mathrm{C}$. Cell lysates were assayed for lactate dehydrogenase (LDH) activity as described previously (Sapolsky et al., 1988). Cellular lysates could be stored at $4^{\circ} \mathrm{C}$ for several days with no 
significant loss of activity. Enzyme activity was measured as a decrease in absorbance (corresponding to the amount of NADH present) at 340 $\mathrm{nm}$ in a Beckman DU-64 spectrophotometer (Sapolsky et al., 1988). LDH is a biologically stable marker whose activity correlates linearly with cell number (Koh and Choi, 1987). We measured residual LDH remaining in undamaged cells (Sapolsky et al., 1988).

Data analysis and statistics. Data are presented as either the average metabolic rate (an average of 5-10 rate determinations prior to experimental manipulation, mean \pm SEM) or as the percentage of the average basal metabolic rate for the cells on a single coverslip, as indicated. Statistical analyses are delineated in Results and figure captions.

\section{Results}

\section{Assessment of microphysiometry for measurement of} metabolic rate in primary hippocampal cultures

The metabolic rate, as measured by the rate of acidification of the medium, was $27.7 \pm 3.7 \mu \mathrm{V} / \mathrm{sec}$ when the total sensing region was filled with confluent cultures of astrocytes, and 114.3 $\pm 10 \mu \mathrm{V} / \mathrm{sec}$ when the sensing region was focused on primarily neuronal clusters in mixed culture (Table 1). These rates represented cellular responses, as cell-free coverslips and coverslips whose cells were lysed and removed with either water or $0.2 \%$ trypsin showed background noise of $1.25 \pm 0.31 \mu \mathrm{V} / \mathrm{sec}$. Further, the metabolic rate was proportional to the percentage of the total sensing field covered by cells (Fig. 2). A specific cellular signal (i.e., above the $95 \%$ confidence interval of the signal from cell-free background) was detectable when as little as $20 \%$ of the $1 \mathrm{~mm}^{2}$ total sensing region was occupied with mixed cultures at the stated plating density (see Materials and Methods).

Pretreatment of cultures with different concentrations of glucose altered the basal metabolic rate (Fig. $3 A$ ). Hyperglycemic conditions significantly enhanced metabolic rate, while $24 \mathrm{hr}$ pretreatment with $0 \mathrm{~mm}$ glucose substantially reduced the metabolic rate. Visual inspection of these cultures revealed an essentially complete loss of overlying neurons in this aglycemic state, although some glia remained. The remaining metabolic rate recorded under these circumstances agreed well with what would be expected from a pure astrocyte culture (see Table 1).

Cyanide $(2 \mathrm{~mm})$ in the perfusate for $2 \mathrm{hr}$ reduced metabolic rate $32 \%$ (Fig. $3 B$ ), indicating the sensitivity of the cultures to

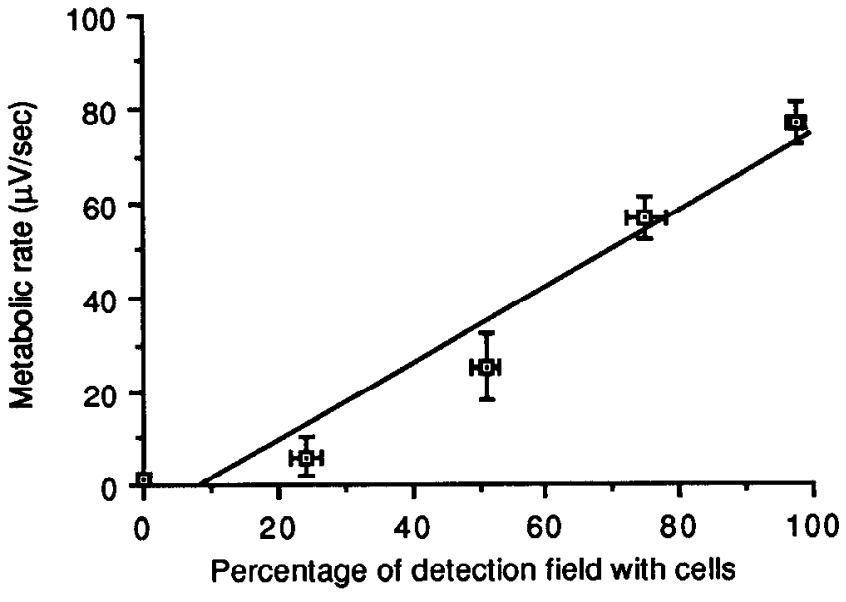

Figure 2. Metabolic rate measurements are proportional to cell density. Mixed hippocampal cultures were plated on coverslips in a concentrated area using a cloning ring. A low-volume flow chamber containing the coverslip was mounted on a microscope stage, and the beam from an He-Ne laser was projected through the optics of the microscope as described by Parce et al. (1989). Acidification rates were thus recorded from cells directly over the $1 \mathrm{~mm}^{2}$ region of the silicon light-activated sensor illuminated by the laser. We could move the microscope stage and thus manipulate the portion of the coverslip from which we gathered metabolic rate data. The cells werc plated in the center of the coverslip, so that a sharp boundary existed between coverslips with and without cells. We moved the stage so that an increasing fraction of the illuminated field contained cells. The microscope stage was moved by predetermined amounts to vary the percentage of the fixed measurement area containing cells. The fraction of the measurement area containing cells was estimated using an ocular grid. Data represent the average metabolic rate determinations for three separate experiments. Linear regression analysis: $r^{2}=0.955 ; p<0.01$.

disruption of oxidative metabolism. Thus, these experiments show that the microphysiometer is sensitive to changes in the metabolic status of mixed cultures. Treatments that increase the supply of glucose increase the metabolic rate in a dose-dependent manner, while treatments that interfere with metabolism decrease the metabolic rate.

Table 1. Effect of kainic acid on metabolic rate in mixed and pure astrocytic cultures

\begin{tabular}{lcccc} 
& $n$ & $\begin{array}{l}\text { Average basal } \\
\text { rate }(\mu \mathrm{V} / \mathrm{sec})\end{array}$ & $\begin{array}{l}\text { Average rate } \\
\text { in treatment } \\
(\mu \mathrm{V} / \mathrm{sec})\end{array}$ & $\begin{array}{c}\text { Percentage } \\
\text { increase in } \\
\text { basal rate }\end{array}$ \\
\hline $\begin{array}{l}\text { Mixed cultures } \\
500 \mu \mathrm{M} \mathrm{KA} \\
500 \mu \mathrm{M} \mathrm{KA}+ \\
\quad 1 \mathrm{mM} \mathrm{KYN} \mathrm{acid}\end{array}$ & 4 & $114.3 \pm 10$ & $191.4 \pm 12^{* * *}$ & $71.4 \pm 9^{a, b}$ \\
$\begin{array}{c}\text { Astrocyte cultures } \\
500 \mu \mathrm{M} \mathrm{KA}\end{array}$ & 4 & $96 \pm 18$ & $104.5 \pm 17$ & $9.8 \pm 2^{b}$ \\
& 7 & $27.7 \pm 3.7$ & $36.6 \pm 5^{* * *}$ & $31.7 \pm 4.9^{a}$
\end{tabular}

Mixed hippocampal cultures (see Materials and Methods) or pure astrocyte cultures (see Materials and Methods) were loaded into microphysiometer chambers and exposed to kainic acid (KA) as described in Figures 2 and 4 . The mixed culture data are presented from clusters of primarily neurons isolated with the laser procedure described in Materials and Methods and in the caption to Figure 2. The astrocytic culture data were obtained from pure astrocyte cultures.

**** Significant $(p<0.001)$ increase in metabolic rate when compared with the basal rate using Student's $t$ test for paired samples. ANOVA comparison of the percentage increase in basal rates across treatments was statistically significant $(p<0.001)$.

${ }^{a}$ Statistically significant difference $(p<0.05)$ between the percentage increase in basal rate of neurons as compared with glial cultures using the Fisher PLSD test.

${ }^{b}$ Statistically significant $(p<0.05)$ difference between the neuronal responses to KA in the absence as compared to the presence of kynurenic acid (KYN) using the Fisher PLSD test. 

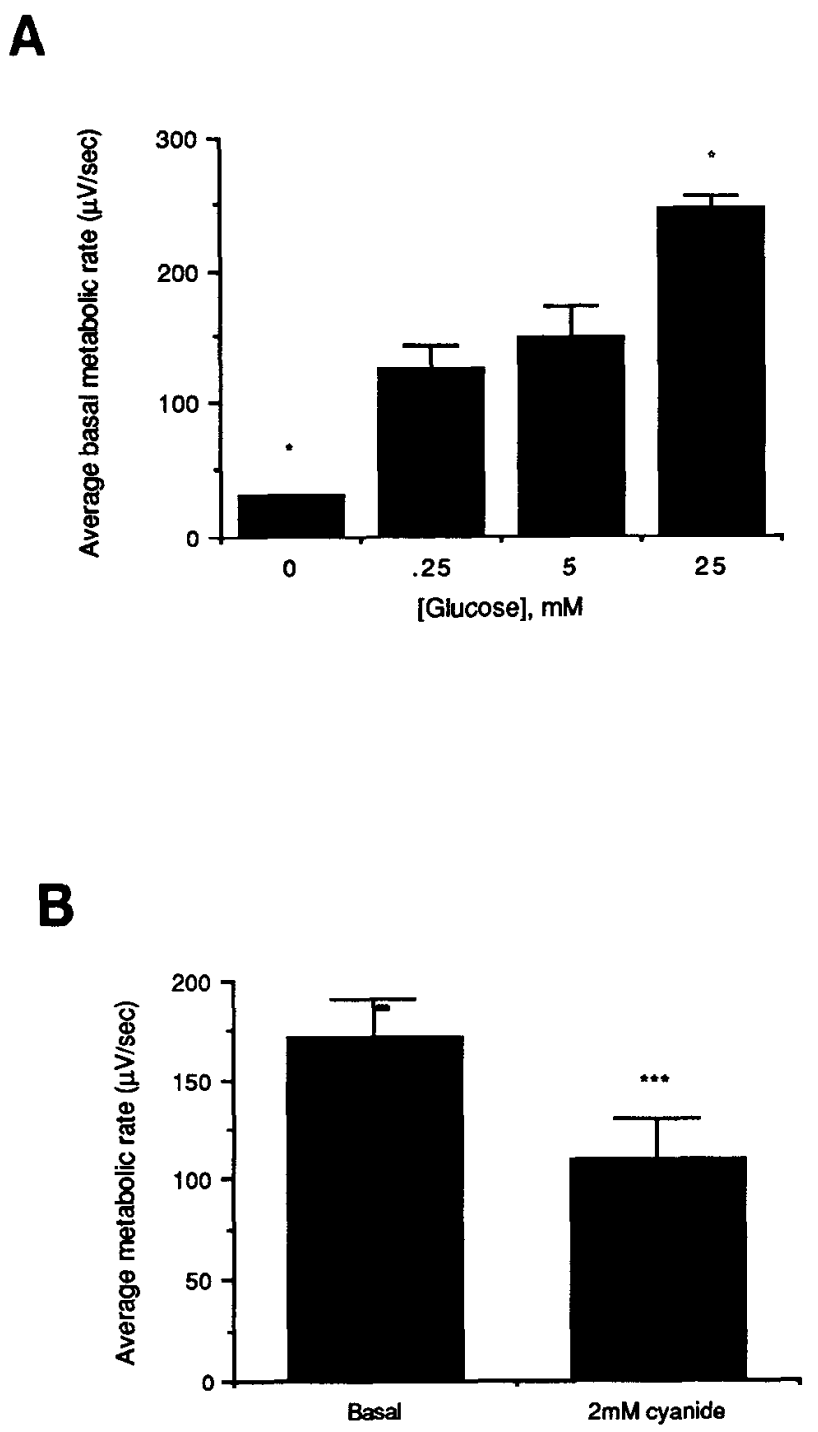

Figure 3. Metabolic rate is sensitive to substrate availability. $A$, Mixed hippocampal cultures were pretreated $24 \mathrm{hr}$ prior to the experiment with DMEM containing different concentrations of glucose $(0,0.25,5$, or $25 \mathrm{mM}$ ). Metabolic rate determinations were made in bicarbonatefree DMEM with the same concentrations of glucose. ANOVA revealed a significant glucose effect $(p<0.0001) .^{*}, p<0.05$ when compared against the normal ( $5 \mathrm{~mm}$ ) glucose group with the Fisher PLSD test. $B$, Cells were acutely exposed to $2 \mathrm{~mm}$ cyanide for $2 \mathrm{hr}$ during the metabolic rate determinations. Rates were comparcd with basal rates using a paired Student's $t$ test $\left.{ }^{* * * *}, p<0.0001\right)$. Data in both figures represent average metabolic rate ( $\mu \mathrm{V} / \mathrm{sec}$; see Materials and Methods) determinations for five to seven separate experiments.

\section{Effects of kainic acid on metabolic rate of hippocampal mixed cultures}

Acute exposure (5-10 min) of mixed cultures to $500 \mu \mathrm{M}$ of kainic acid increased metabolic rate (Fig. 4, Table 1). Upon removal of the kainic acid, the metabolic rate returned to basal levels. Repeated exposures evoked similar changes in metabolic rate. With more prolonged kainic acid exposure, the metabolic response eventually began to wane (Fig. 4). The response included both neuronal and glial components, as pure astrocyte cultures were also responsive to the drug (Table 1). Parallel experiments (using the laser setup described in the caption to Fig. 2, with

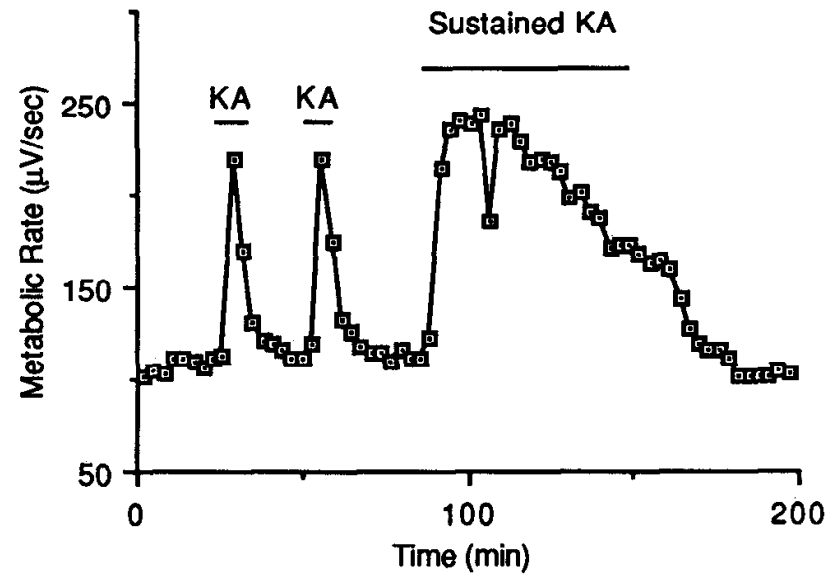

Figure 4. Kainic acid increases metabolic rate. Coverslips containing mixed hippocampal cultures were loaded into chambers of the microphysiometer. Regions of predominantly neurons were focused upon using the laser setup described in Materials and Methods and the caption to Figure 2. A steady basal metabolic rate was determined for the first $30 \mathrm{~min}$ to $1 \mathrm{hr}$ after chamber assembly. Where indicated, a $500 \mu \mathrm{M}$ kainic acid $(K A)$ solution (in bicarbonate-free DMEM, pH 7.4) was injected into the chambers using a $750 \mu \mathrm{l}$ injection loop. Measurements in response to acute (3-5 min) exposures were obtained, and the solution was switched to normal bicarbonate-free DMEM lacking the drug. Sustained exposure to kainic acid was obtained by switching the bathing solution. Data are representative of three similar experiments.

which it is possible to focus on isolated clusters of neurons, relatively free from the presence of glia) indicated that neurons were more responsive to kainic acid than glia (Table 1).

The response to kainic acid appeared glutamate receptor mediated, as it was prevented by $1 \mathrm{~mm}$ kynurenic acid (Fig. 5, Table 1). It is important to note that $1 \mathrm{~mm}$ kynurenic acid blocks all subtypes of the glutamate receptor (Ganong et al., 1983). Thus, these results do not distinguish between a direct action of kainic acid on the kainate receptor subtype to increase metabolic rate or a secondary effect from depolarization and subsequent activation of additional glutamate receptor subtypes (such as the NMDA receptor). Finally, the metabolic response to kainic acid was dose dependent (Fig. 6), with an estimated $\mathrm{EC}_{50}$ of $\sim 100 \mu \mathrm{M}$ in this paradigm.

Overstimulation of glutamate receptors, including the kainic acid receptor, is neurotoxic (Coyle et al., 1981; Rothman et al., 1987; Frandsen et al., 1989). Presumably, such overstimulation produces ion imbalances with which the cell cannot cope because energy-dependent ion homeostatic mechanisms are not fueled sufficiently with ATP. These ideas predict that overstimulation of kainic acid receptors should produce an initial stimulation of cellular metabolism, which the cells cannot maintain; this was observed when cells were exposed continuously to a toxic dose (2.5 mM) dose of kainic acid for up to $8 \mathrm{hr}$ (Fig. 7).

Because a sustained exposure to kainic acid is a rather severe and unphysiological insult, we next exposed cells to a $10 \mathrm{~min}$ pulse of $2.5 \mathrm{~mm}$ kainic acid (an exposure that is still toxic) and allowed cells to recover in normal perfusate lacking the drug (Fig. 8). At $5 \mathrm{hr}$ recovery, when the metabolic rate was significantly decreased relative to baseline (Fig. 8), LDH measurements indicated no significant loss of cell viability relative to unexposed cultures (Fig. 8 inset). Thus, a decrease in metabolism appcars to precede measurable cell loss. 


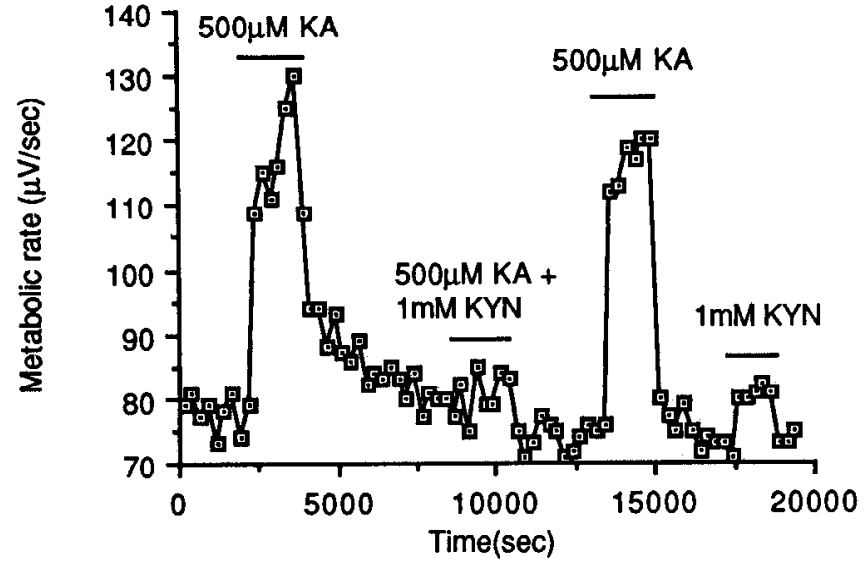

Figure 5. The kainic acid-induced increase in metabolic rate is receptor mediated. Coverslips containing mixed hippocampal cultures were loaded into chambers of the microphysiometer, and a steady basal metabolic rate from predominantly neurons (see the caption to Figure 4) was determined. Where indicated, a $500 \mu \mathrm{m}$ kainic acid $(K A)$ solution (pH 7.4) in the presence or absence of $1 \mathrm{~mm}$ kynurenic acid $(K Y N)$, a glutamate receptor antagonist, was injected using injection loops of 750 $\mu \mathrm{l}$ vol. Several measurements were obtained, and the solution was returned to normal bicarbonate-free DMEM lacking drugs. Kynurenic acid at $1 \mathrm{~mm}$ had only a very small effect (see Table 1) on metabolic rate. Data shown are representative of three separate experiments.

\section{Discussion}

\section{Application of silicon microphysiometry to neurobiology}

We used a silicon microphysiometer to measure the metabolic rate of mixed hippocampal cultures. Our data indicate some of the advantages and likely limitations of this technology when applied to neuronal cultures. Previous studies have shown that this device, which measures the rate of acidification of the extracellular perfusate, provides a sensitive index of overall cellular metabolic rate; the acidification rate is proportional to lactate production and oxygen consumption (Parce et al., 1989; Owicki et al., 1990). In this study, we demonstrated the sensitivity of this device for use with primary hippocampal cultures. Basal metabolic rates far above background could be detected in pure astrocytic cultures and in detection fields filled primarily with neurons. The neuronal metabolic rate was approximately four times higher than the astrocytic rate (this is likely to be an underestimate because of the presence of some astrocytes in the primarily neuronal fields, and the fact that such neuron-rich clusters did not fill the total sensing region). The smaller rate in astrocyte cultures compared with neuronal clusters could reflect a different cell density, as glia are flattened and take up a greater surface area on the coverslips than do the neurons. It was not possible in this study to determine metabolic rate on a per cell basis; a more detailed analysis of these differences is under way. The rate of acidification of the external medium was proportional to the size of the cell-containing field overlying the lightaddressable potentiometric sensor, and the rate represents cellular acid extrusion, as the rate was abolished when the cells were lysed.

The acidification rate comprises a summation of all cellular processes involving acid extrusion. In most cells, this measure would indicate lactate and $\mathrm{CO}_{2}$ production/extrusion, proton production/extrusion from ATP hydrolysis, and proton extrusion mechanisms unrelated to energy metabolism. Previous work

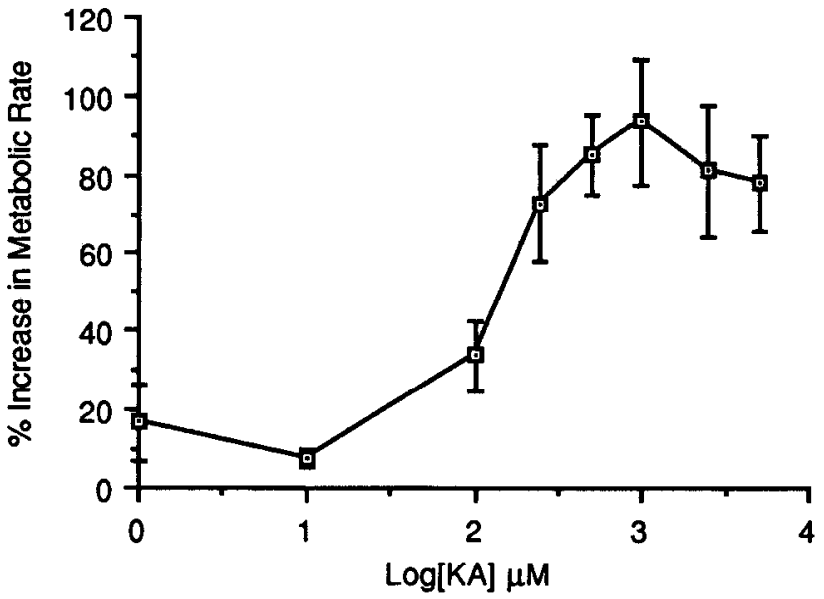

Figure 6. The kainic acid-induced increase in metabolic rate is dose dependent. Mixed hippocampal cultures were exposed to sequential (from lowest to highest concentrations) injections of different concentrations of kainic acid ( $K A ; \mathrm{pH} 7.4)$. Several measurements at each concentration were obtained, and the cells were allowed to recover in normal bicarbonate-free DMEM until a steady basal rate was again obtained between each injection of the drug. Responses were calculated as the percentage of the basal metabolic rate for each coverslip and presented versus the log of the kainic acid concentration. Data shown are the average of $n=6$ similar experiments.

with other cell types demonstrated that the acidification rate was quite similar to the rate of $\mathrm{O}_{2}$ consumption and the rate of lactate production (Parce et al., 1989). Those data indicated that the acidification rate includes both aerobic and anaerobic metabolism components. Indeed, our results with cyanide, used at

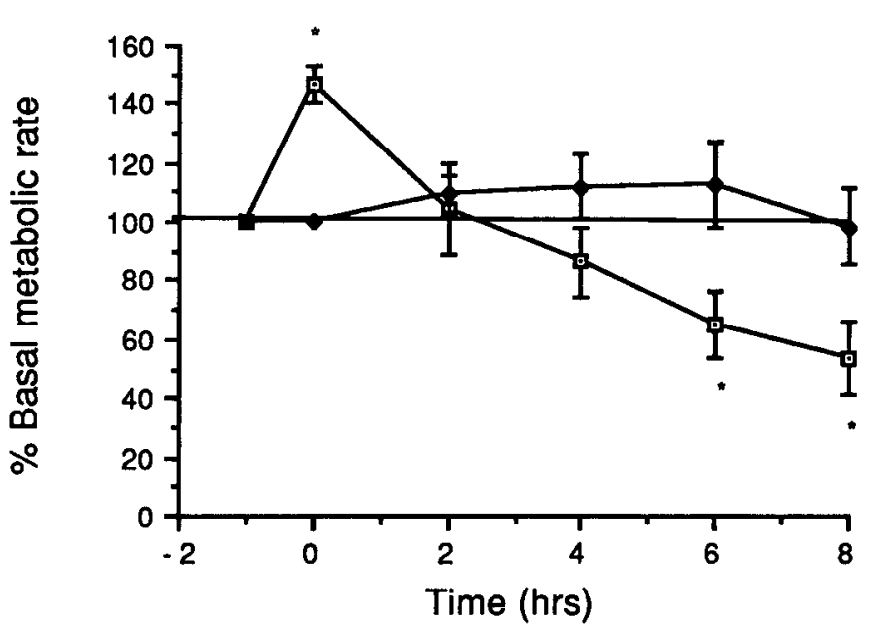

Figure 7. Effect of sustained exposure to kainic acid. Mixed hippocampal cultures were exposed to $2.5 \mathrm{~mm}$ kainic acid (pH 7.4) beginning at $t=0 \mathrm{hr}$. The average metabolic rate was determined at the times indicated and presented as a percentage of the average basal metabolic rate (see Materials and Methods). Data represent the averages of $n=8$ separate experiments. Average basal metabolic rate was $160.6 \pm 18 \mu \mathrm{V} /$ sec. Open symbols indicate mixed cultures exposed to $2.5 \mathrm{~mm}$ kainic acid at $t=0$, and the solid symbols indicate control cultures not exposed to kainic acid. Two-factor ANOVA revealed a significant effect of kainic acid over time, when compared with untreated cultures $(p \leq 0.0005)$. The asterisk indicates a significant difference between the response to kainic acid and the basal metabolic rate $(p<0.05)$ with the Fisher PLSD test. There was no significant effect of time on metabolic rate in untreated cultures. 


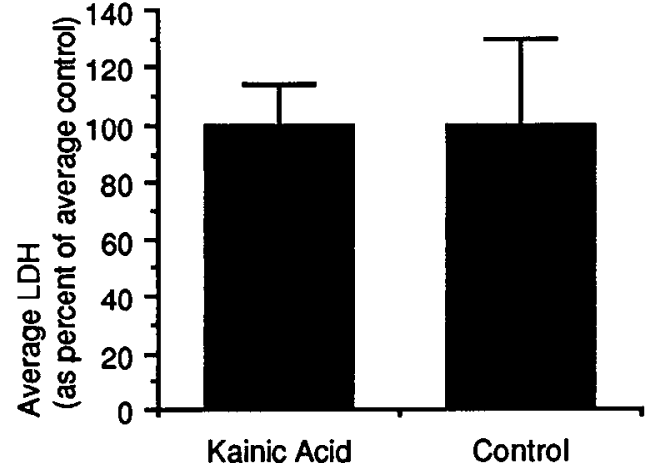

inset

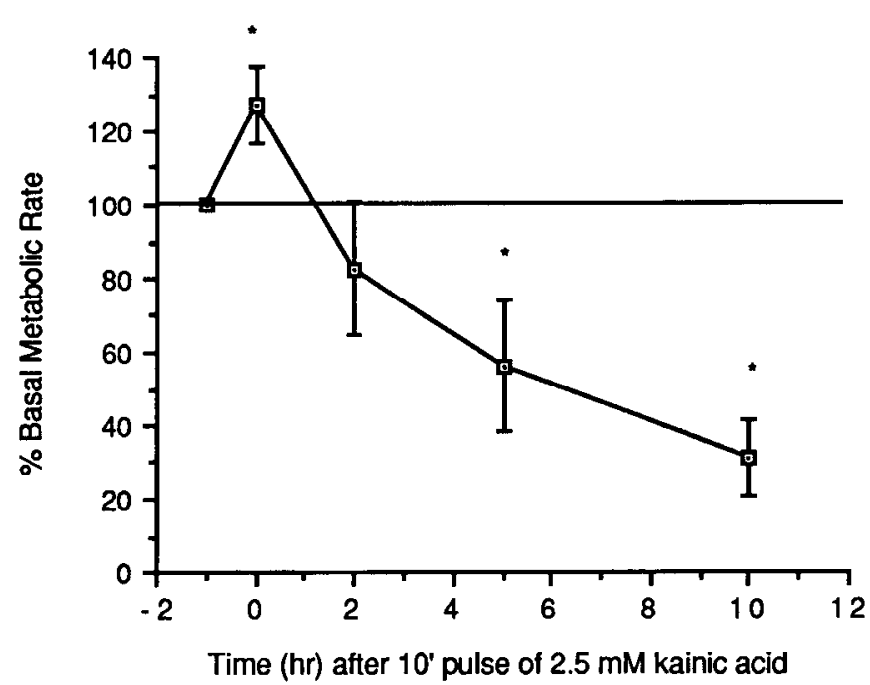

Figure 8. Effect of $10 \mathrm{~min}$ exposure to $2.5 \mathrm{~mm}$ kainic acid. Mixed hippocampal cultures were exposed to $2.5 \mathrm{~mm}$ kainic acid (pH 7.4) for $10 \min$ at $t=0$ and then returned to normal perfusion medium for the recovery times indicated. Data represent the average of $n=3$ separate experiments. Data are presented as the average metabolic rate $(\mu \mathrm{V} / \mathrm{sec})$; see Materials and Methods. Average basal metabolic rate was $213.3 \pm$ $29 \mu \mathrm{V} / \mathrm{sec}$. ANOVA revealed a significant decline in metabolic rate $(p$ $<0.0037)$. The asterisk indicates statistical significance $(p<0.05)$ when compared with basal rates $(t=-1 \mathrm{hr})$ using Fisher PLSD test. Inset, Cultures were exposed to $2.5 \mathrm{mM} \mathrm{KA}$ or vehicle (DMEM) for $10 \mathrm{~min}$ as described above, and at $5 \mathrm{hr}$ after the exposure, coverslips were removed and prepared for LDH determination as described in Materials and Methods. $\mathrm{LDH}$ values are presented relative to the average control value in $n=6-8$ separate experiments. No statistical difference when compared using Student's $t$ tcst for unpaircd samples.

a concentration sufficient to interfere maximally with oxygendependent metabolism (Olesen, 1986), indicate that a proportion, but by no means all, of the acidification rate represents aerobic metabolism in hippocampal mixed cultures. The actual contribution of aerobic metabolism to the metabolic rate measured in this study may be an underestimate because anaerobic metabolism may have been stimulated to compensate for the cyanide-induced loss of oxidative ATP production. Thus, from this study, the actual percentage of the measurement that is lactate production is unknown for hippocampal mixed cultures.

ATP hydrolysis produces protons, which are extruded by several membrane transport mechanisms (Roos and Boron, 1981), including the $\mathrm{Na}^{+} / \mathrm{H}^{+}$exchanger in hippocampal neurons (Raley-Susman et al., 1991). There are additional, $\mathrm{HCO}_{3}$-dependent mechanisms; however, the nominally $\mathrm{HCO}_{3}$-free perfusion medium precludes the contribution of these transport mechanisms. We have shown previously that in nominally $\mathrm{HCO}_{3}$-free solutions, the primary mechanism of acid extrusion is an $\mathrm{Na}^{+} / \mathrm{H}^{+}$ exchanger (Raley-Susman et al., 1991). Therefore, an important consideration of the metabolic rate measurement in this study is the effect of neurotransmitters and hormones on this acid extruder. For example, direct activation of the $\mathrm{Na}^{+} / \mathrm{H}^{+}$antiport would produce an apparent increase in metabolic rate using the microphysiometer. Indeed, growth factors, phorbol esters, and various receptor agonists have been shown in other cell types to activate $\mathrm{Na}^{+} / \mathrm{H}^{+}$exchange activity (Moolenaar, 1986; Grinstein et al., 1989). While such an effect cannot be ruled out in the present study, glutamate analogs transiently $(30 \mathrm{sec})$ decreased intracellular $\mathrm{pH}$, when measured with a fluorescent $\mathrm{pH}$ indicator; this effect was very brief and presumably reflects direct proton influx via channel opening, or decreased activity of the $\mathrm{Na}^{+} / \mathrm{H}^{+}$exchanger (K. M. Raley-Susman, unpublished observations). Thus, the extracellular acidification rates, occurring during and after the brief effect on intracellular $\mathrm{pH}$, most probably represent a true increase in metabolic rate.

Another important consideration in this study is the effect of conditions in the microphysiometer on mixed hippocampal cultures. Silicon microphysiometry necessitates the use of solutions with very low buffering capacity $\left(1 \mathrm{mM} \mathrm{PO}_{4}\right.$, nominally bicarbonate free) in order to detect sensitively acidification of the medium due to cellular acid extrusion. Although the cells do not appear to be extremely sensitive to low bicarbonate concentrations (Raley-Susman et al., 1991), the long-term effects of such low-buffering-capacity solutions are unknown and may affect the normal response of neurons and glia. In fact, in contrast to other cell types (Wada et al., 1991), we found that metabolic rate in control cultures began to decrease after $10 \mathrm{hr}$ or more of constant perfusion with low buffering medium. This decreased rate could reflect cell loss or hampered metabolism resulting from prolonged time periods in low buffering medium or from the shear stress $\left(1 \mathrm{dyne} / \mathrm{cm}^{2}\right)$ caused by the perfusion. Although this shear stress causes minimal changes in some cell types (Levesque et al., 1989), the effects on neuronal cultures are unknown. Further work is ongoing to characterize more fully the effects of low buffering medium and perfusion shear force in hippocampal mixed cultures.

Thus, secondary measurements of cell viability, such as LDH determination, are necessary to distinguish between a true decrease in metabolic rate and a decrease resulting from loss of cclls. However, the advantages afforded by the sensitive, timeintegrated measurements of metabolism enable increased time resolution of metabolic events evoked by neurotransmitter activation.

\section{Effects of kainic acid on cellular metabolism}

This is the first direct demonstration that kainic acid increases metabolic rate $(\sim 80 \%)$ in hippocampal mixed cultures in a dosedependent and receptor-mediated manner. This increase most likely reflects the depolarization-induced activation of energydependent ion extrusion pumps, since kainic acid has been shown 
to induce changes in $\mathrm{Na}^{+}, \mathrm{K}^{+}, \mathrm{H}^{+}$, and $\mathrm{Ca}^{2+}$ fluxes (Biziere and Coyle, 1978; Lothman and Collins, 1981; Ben-Ari, 1985; Lazarewicz et al., 1986; Kobayashi et al., 1990). The estimated $\mathrm{EC}_{30}$ for kainic acid in this study $(100 \mu \mathrm{M})$ agrees quite well with that for electophysiological responses to excitatory amino acid exposure in culture (Koroshetz et al., 1990). Pure astrocytic cultures also exhibited an increased metabolic rate upon exposure to kainic acid, although the increase was less than half the magnitude of that seen with primarily neuronal clusters. Astrocytes in vitro express kainic acid receptors (Backus et al., 1989). The difference in the intensity of the metabolic response to kainic acid is intriguing and could relate to differences in receptor density or to differences in sensitivity to the excitatory amino acid (amount of depolarization, changes in calcium, etc.). Further, the responsiveness of glia to kainic acid in mixed culture could be modified indirectly by the presence of neurons.

Early work suggested that neurons expend more than half of their ATP for the maintenance of ion homeostasis (Siesjo, 1978). Thus, activation of kainic acid receptors, leading to influx of $\mathrm{Na}^{+}$, would be expected to activate energy-dependent ion extruders, such as the $\mathrm{Na}^{+} / \mathrm{K}^{+}$ATPase and $\mathrm{Na}^{+} / \mathrm{Ca}^{2+}$ exchange mechanism, and subsequently activate cellular metabolism. With increasing doses of kainic acid, the depolarization of neurons would be sufficient to open voltage-dependent calcium channels, further activating ion extruders. The large increase in metabolism is quite striking and suggests that, under conditions of energy failure, the brain's ability to withstand neurotransmitter activation could be altered.

In the present study, we used a concentration of kainic acid that has been shown to be neurotoxic in other in vitro studies (Frandsen et al., 1989; Galarraga et al., 1990) and continuously monitored the metabolic response to this exposure. We found that, following the initial metabolic excitation, metabolic impairment began as little as $4 \mathrm{hr}$ after a brief $(10 \mathrm{~min})$ or a continuous exposure to kainic acid. At these early times, the decreased metabolic rate preceded measurable cell lysis, based on LDH measurements. Thus, the decline in rate most likely represents a true decrease in metabolic rate. At later time points, LDH measurements also declined, indicating measurable cell lysis contributing to the decline in metabolic rate. Is the decline in metabolic rate (extracellular acidification) a reflection of decreased energy (ATP) availability or a decreased cellular demand for cncrgy? In vivo studics have demonstrated that excitotoxin exposure produces a sustained increase in electrophysiological excitability (McGregor et al., 1990), increased oxygen and glucose utilization (Franck et al., 1986; Ingvar, 1986; Siesjo et al., 1986), and a marked decrease in ATP concentration (Ingvar, 1986). Similarly, excitotoxins such as kainic acid cause a decrease in ATP levels in striatal slices (Biziere and Coyle, 1978). While these parameters have not been measured in primary hippocampal cultures over the time course utilized in this study, this literature suggests that the decrease in metabolic rate would seem to be secondary to a decline in substrate availability, rather than to decreased cellular demand. This scenario is certainly consonant with current thinking regarding the role of energy depletion in excitotoxin-induced neuron death.

Another important finding mentioned above is that the measured decrease in metabolic rate, whether because of decreased demand or decreased supply, preceded cell death, as assessed by LDH measurement. These data suggest that metabolic impairment is detectable soon after excitotoxin exposure and may serve as a sensitive index of neurotoxicity. While we have not yet shown conclusively that this is the case, earlier in vivo studies have demonstrated that the indices of hypermetabolism and substrate depletion precede overt cell degeneration (Franck et al., 1986; Ingvar, 1986; Siesjo et al., 1986; Woolf, 1987; McGregor et al., 1990).

In summary, we have shown the feasibility of using novel silicon microphysiometry for assessing metabolism in brain cultures. Given the caveats discussed, the sensitivity of this approach and its capacity to provide real-time data suggest a broad array of potential uses. Microphysiometry affords the advantage of being able to couple continual time-integrated monitoring of metabolic rate with measurements of toxicity and/or substrate availability, in order to test hypotheses of the relationships among these variables. In a first application of this technology, we have demonstrated that an excitotoxin initially stimulates metabolic rate in hippocampal mixed cultures (in a dose-dependent, receptor-mediated manner) and then causes a decline in metabolism that precedes cell loss. These results delineate a timewindow for the onset of excitotoxin-induced damage in vitro.

\section{References}

Backus KH, Kettenmann H, Schachner M (1989) Pharmacological characterization of the glutamate receptors in cultured astrocytes. J Neurosci Res 22:274-282.

Banker G, Cowan W (1977) Rat hippocampal neurons in dispersed cell culture. Brain Res 126:397-425.

Ben-Ari Y (1985) Limbic seizure and brain damage produced by kainic acid: mechanisms and relevance to human temporal lobe epilepsy. Neuroscience 14:375-403.

Biziere K, Coyle JT (1978) Effects of kainic acid on ion distribution and ATP levels of striatal slices incubated in vitro. J Neurochem 31: 513-520.

Choi DW (1988) Glutamate neurotoxicity and diseases of the nervous system. Neuron 1:623-634.

Coyle JT, Bird SJ, Evans RH, Gulley RL, Nadler JV, Nicklas WJ, Olney JW (1981) Excitatory amino acid neurotoxins: selectivity, specificity, and mechanism of action. Neurosci Res Prog Bull 19:329-427.

Cremer JE, Seville MP, Cunningham VJ (1988) Tracer 2-deoxyglucose kinetics in brain regions of rats given kainic acid. J Cereb Blood Flow Metab 8:244-253.

Franck G, Sadzot B, Salmon E, Depresseux JC, Grisar T, Peters JM, Guillaume M, Quaglia L, Delfiore G, Lamotte D (1986) Regional cerebral blood flow and metabolic rates in human focal epilepsy and status epilepticus. Adv Neurol 44:935-948.

Frandsen A, Drejer J, Schousboc A (1989) Direct cvidence that excitotoxicity in cultured neurons is mediated via $N$-methyl-D-aspartate (NMDA) as well as non-NMDA receptors. J Neurochem 53:297-299.

Galarraga E, Surmeier DJ, Kitai ST (1990) Quinolinate and kainate neurotoxicity in neostriatal culture is potentiated by co-culturing with neocortical neurons. Brain Res 512:269-276.

Ganong AH, Lanthorn TH, Cotman CW (1983) Kynurenic acid inhibits synaptic and acidic amino acid-induced responses in the rat hippocampus and spinal cord. Brain Res 273:170-174.

Grinstein S, Rotin D, Mason MJ (1989) $\mathrm{Na} / \mathrm{H}$ exchange and growth factor-induced cytosolic $\mathrm{pH}$ changes: role in cellular proliferation. Biochim Biophys Acta 988:73-97.

Horner HC, Packan D, Sapolsky RM (1990) Glucocorticoids inhibit glucose transport in cultured hippocampal neurons and glia. Neurocndocrinology 52:57-64.

Ingvar M (1986) Cerebral blood flow and metabolic rate during seizures. Ann NY Acad Sci 462:194-206.

Kobayashi S, Kikuchi H, Ishikawa M, Hashimoto K (1990) Regional changes of tissue $\mathrm{pH}$ and ATP content in rat brain following systemic administration of kainic acid. Brain Res 514:352-354.

Koh JY, Choi DW (1987) Quantitative determination of glutamate mediated cortical neuronal injury in cell culture by lactate dehydrogenase efflux assay. J Neurosci Methods 20:83-90.

Koroshetz WJ, Freese A, Difiglia M (1990) The correlation between excitatory amino acid-induced current responses and excitotoxicity in striatal cultures. Brain Res 521:265-272.

Lazarewicz JW, Lehmann A, Hagberg H, Hamberger A (1986) Effects 
of kainic acid on brain calcium fluxes studied in vivo and in vitro. $\mathrm{J}$ Neurochem 46:494-498.

Levesque JM, Sprague EA, Schwartz CJ, Nerem RM (1989) The influence of shear stress on cultured vascular endothelial cells: the stress response of an anchorage-dependent mammalian cell. Biotech Prog 5:1-8.

Lothman EW, Collins RC (1981) Kainic acid induced limbic seizures: metabolic, behavioural, electroencephalographic and neuropathological correlates. Brain Res 218:299-318.

McGregor IS, Menendez JA, Atrens DM (1990) Metabolic effects obtained from excitatory amino acid stimulation of the sulcal prefrontal cortex. Brain Res 529:1-6.

Moolenaar WH (1986) Regulation of cytoplasmic $\mathrm{pH}$ by $\mathrm{Na} / \mathrm{H}$ exchange. Trends Biochem Sci 11:141-143.

Nishizaki T, Yamauchi R, Okada Y (1988) Enhancement of the oxygen consumption in the hippocampal slices of the guinea pig induced by glutamate and its related substances. Neurosci Lett 85:61-64.

Novelli A, Reilly JA, Lysko PG, Henneberry RC (1988) Glutamate becomes neurotoxic via the $N$-methyl-D-aspartate receptor when intracellular energy levels are reduced. Brain Res 451:205-212.

Olesen SP (1986) Rapid increase in blood-brain barrier permeability during severe hypoxia and metabolic inhibition. Brain Res 368:2429.

Owicki JC, Parce JW (in press) Biosensors based on the energy me- tabolism of living cells: the physical chemistry and cell biology of extracellular acidification. Biosens Bioeng, in press.

Owicki JC, Parce JW, Kercso KM, Sigal GB, Muir VC, Venter JC, Fraser CM, McConnell HM (1990) Continuous monitoring of receptor-mediated changes in the metabolic rates of living cells. Proc Natl Acad Sci USA 87:4007-4011.

Parce JW, Owicki JC, Kercso KM, Sigal GB, Wada HG, Muir VC, Bousse LJ, Ross KL, Sikic BL, McConnell HM (1989) Detection of cell-affecting agents with a silicon biosensor. Science 246:243-247.

Raley-Susman KM, Cragoe EJJ, Sapolsky RM, Kopito RR (1991) Regulation of intracellular $\mathrm{pH}$ in cultured hippocampal neurons by an amiloride-insensitive $\mathrm{Na}^{+} / \mathrm{H}^{+}$exchanger. J Biol Chem 266:2739-2745.

Roos A, Boron WF (1981) Intracellular pH. Physiol Rev 61:296-434.

Rothman SM, Thurston JH, Hauhart RE (1987) Delayed neurotoxicity of excitatory amino acids in vitro. Neuroscience 22:471-480.

Sapolsky RM, Packan DR, Vale WW (1988) Glucocorticoid toxicity in the hippocampus: in vitro demonstration. Brain Res 453:367-371. Siesjo BK (1978) Brain metabolism. New York: Wiley.

Siesjo BK, Ingvar M, Wieloch T (1986) Cellular and molecular events underlying epileptic brain damage. Ann NY Acad Sci 462:207-223.

Wada HG, Owicki JC, Parce JW (1991) Cells on silicon: bioassays with a microphysiometer. Clin Chem 37:600-601.

Woolf CJ (1987) Excitatory amino acids increase glycogen phosphorylase activity in the rat spinal cord. Neurosci Lett 73:209-214. 